\title{
Über einen allgemeinen Übungsbegriff bei verschiedenen Unterrichtsmethoden in der Planung des Mathematikunterrichtes Konsequenzen für die Übungsforschung
}

\author{
Gabriella Ambrus
}

Abstract. Practice is important in the education of mathematics but is neclected in the didactic of mathematics. One of the reasons is that practice is often defined too "narrowly" and the definitions of practice have in most cases an obscure background theory. In the article a general definition of practice is given, which - in contrast to the usual definitions - views practice from the point of the pupils (practice means activity of pupils). By utilising this definition consequences will be drawn. These consequences serve as for the more exact planning of practice in education as for the analysis of the dependency of practice from teachingsmethods.

In the second part an example will be presented for planning together practice and lesson, in two different teachingsmethods (traditionel, problemsolving). The analysis of both worksheets (one for each method, identical teachingsmaterial) was made on the basis of authors practise in lessons i.e. her own concepts and the experience with pupils at a class 5 . On the basis of the expectable solutions is specified - using a criteriacatalogwhat was practised.

The analysis of practice leads further to the examination of above mentioned dependency from teachingsmethods.

Key words and phrases: didactics of mathematics, practice, lecture planning.

ZDM Subject Classification: D40, F40, U60. 


\section{1. Übungsauffassungen}

Üben ist wichtig. Diese Behauptung wurde niemals für den Unterricht bezweifelt. Schon bei Comenius ist zu lesen, dass eine fortwährende Übung notwendig ist, aber dies „geschickt" gemacht werden soll, und zwar so, dass das Üben zum Verstehen der Inhalte und nicht zum sinnlosen Einprägen führt (Comenius [3]).

Die Praxis des Übens betont(e) aber meistens mechanisches Einüben. Trotz kritischer Positionen gegenüber dieser Praxis während der Reformen des schulischen Unterrichtes seit Ende des XIX. Jahrhundert wurde „Üben“ sowohl in der pädagogischen als auch in der fachdidaktischen Literatur theoretisch wenig diskutiert.

Es ist festzustellen, dass in der pädagogischen Literatur die beschriebenen Übungsauffassungen von Hintergrundtheorien abhängen, welche aber nicht oder nur teilweise beschrieben sind.

Die Übungsauffassungen in der mathematikdidaktischen Literatur gehören zu verschiedenen Begriffsbereichen und können daher einander eher ergänzen. Nach einer ungarischen Auffassung bedeutet „Üben“ das Anwenden von erworbenen Kenntnissen, und es werden auch verschiedene Schwierigkeitsstufen für Üben angegeben (Iker/Szerencsi/Vörös, [9]). In der deutschsprachigen Literatur gibt es meistens zwei Typen von Übungsauffassungen:

- Übung im engen Sinne

- Übung im weiteren Sinne.

Die „engen“ Definitionen konzentrieren sich auf die kognitiven Aspekte des Übens; Üben heißt Entwicklung der Fertigkeiten, Einprägen von Kenntnissen, das Herausbilden von Fähigkeiten und manchmal auch Entwicklung bestimmter heuristischer Strategien zum Lösen von Aufgaben (vgl. Maier [10], Wittmann [16]).

Bei den „,weiteren/erweiterten“ Definitionen kommen auch affektive Aspekte hinzu, wie z.B.: Verhaltensweisen üben, Gewohnheiten üben (vgl. Walsch/Jungk [14], Flade/Goldberg [5], Flade/Pruzina [6], Claus [2], Winter [15]).

Üben wird auch in Bezug auf „Festigen“, ,Vertiefen“, „,Systematisieren“, „Anwenden" und "Wiederholen" beschrieben bei Walsch/Jungk [14], Engelmann/ Liesenberg [4] (wobei Festigen als Überbegriff erscheint) und zwar etwa folgendermaßen: 


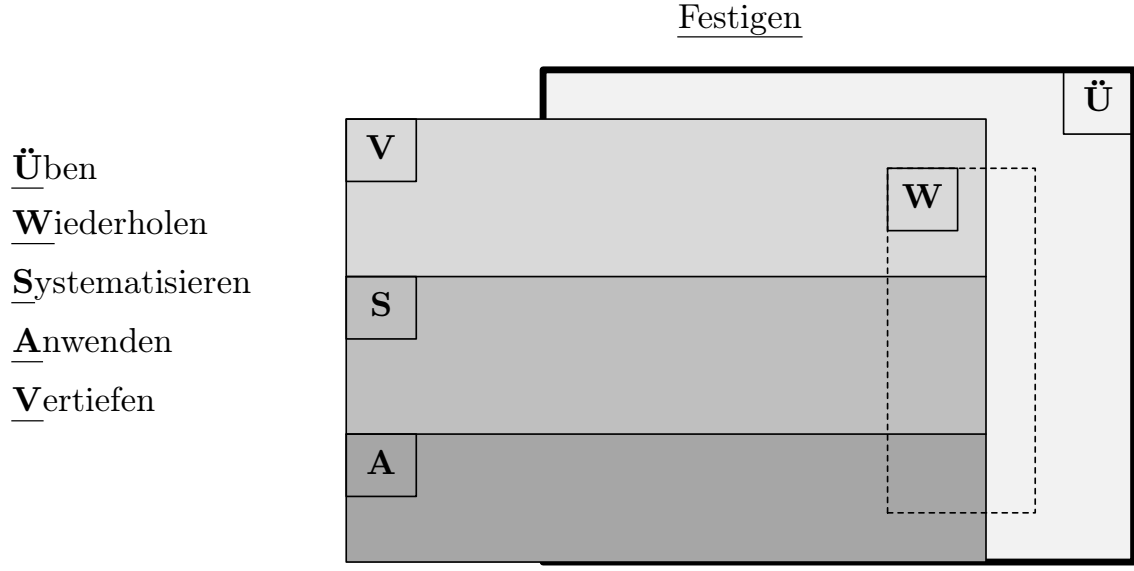

Abbildung 1

Die möglichen Überlappungen der Elemente „Vertiefen“, „Systematisieren“ und "Anwenden“ wurden nicht beachtet. Die „Sonderstellung“ des Wiederholens wird dadurch ausgedrückt, dass dies alle anderen Festigungsformen durchdringt.

Es muss bemerkt werden, dass diese Auffassung des Übens und der erwähnten Bezeichnungen in der mathematikdidaktischen Literatur nicht verbreitet ist.

In den verschiedenen Unterrichtsmethoden (sogar nnerhalb einer Hauptmethode - z.B. nach der Übungsauffassungen von Wittenberg, Wagenschein und Freudenthal in der genetischen Methode (vgl. Ambrus [1]) wird Üben verschiedenerweise beschrieben.

Es scheint so, dass es mehrere Arten des „Übens“ gibt, je nachdem, durch welche Eigenschaften der relevante Unterricht charakterisiert wird.

Wenn aber das Gemeinsame in den Übungsbeschreibungen betrachtet wird, kann ein allgemeiner Übungsbegriff angegeben werden, der auch als Ausgang für eine Erforschung des Übens dienen kann.

\section{Allgemeiner Übungsbegriff}

Üben bedeutet Schüleraktivität. Diese Schüleraktivität bezieht sich auf das Erwerben von mathematischen Kenntnissen und auf das Ausbilden von Fähigkeiten, die zum Erwerben von mathematischen Kenntnissen notwendig sind. 
Daraus folgt einerseits die weite Auffassung des Übens, andererseits dass das Üben nicht nur nach dem Erlernen erfolgt, sondern dass in allen Lernphasen geübt wird. Es ist also weiterhin festzustellen:

- Üben als Schüleraktivität kann nur in konkreten Unterrichtssituationen betrachtet werden.

- Üben durchdringt den ganzen Lernprozess, und es wird nicht nur mit den sogenannten Übungsaufgaben (nach dem Erarbeiten) geübt.

- Da im Lehrplan das Erwerben von mathematischen Kenntnissen, Algorithmen, Denkweisen, Haltungen, Einstellungen vorgeschrieben ist (was durch Schüleraktivität geschieht), muss bei einer Untersuchung des Übens mindestens das betrachtet werden, was von diesen geübt wird.

Es wird im weiteren - anhand der Planungstätigkeit des Lehrers in verschiedenen Unterrichtsmethoden - untersucht, welche Konsequenzen diese neue Definition für den Unterricht hat. Diese Untersuchung ermöglicht auch eine konkrete vergleichende theoretische Analyse der Methodenabhängigkeit des Übens.

\section{Untersuchung des Übens}

\subsection{Theoretische Grundlagen, Rahmenbedingungen}

Diese Untersuchung wurde mit Hilfe von vier ungarischen Unterrichtsbeispielen durchgeführt. Die Sachverhalte für die Arbeitsblätter wurden so ausgewählt, dass möglichst viele Bereiche der Mathematik (Arithmetik, Zahlentheorie, Geometrie), verschiedene Schulstufen $(5,6,7)$ und verschiedene Typen des Bearbeitens (Begriffsbildung, innermathematische Anwendung, Erarbeiten von Definitionen und Eigenschaften) betrachtet werden.

Für die Planung dieser Beispiele haben wir Arbeitsblätter mit zugehörigen Kommentaren verfasst und verwendet. In den Kommentaren wurden nicht nur die Lösungen, sondern auch die erwarteten Schülerlösungen (Erwartungen) angegeben. Dies ermöglicht, zu bestimmen, was konkret bei den einzelnen Aufgaben geübt wurde, und damit das Üben inhaltlich vergleichbar ist, wurden Kriterien angegeben: Kenntnisse und Allgemeine Erfordernisse aus dem Ungarischen Lehrplan und Grundtätigkeiten aus dem Österreichischen Lehrplan. Der ergänzende Charakter der Erfordernisse und Grundtätigkeiten erklärt, warum beide Anforderungskataloge nötig sind (vgl. Ambrus [1]). 
Der Grund der Auswahl der Kriterien ist, dass das Üben - schulisches Üben - das Erwerben von verschiedenen Kenntnissen und Fähigkeiten bedeutet, die in den NAT-Lehrplänen vorgeschrieben sind. Daraus folgt auch, dass für die Charakterisierung des Übens auch andere Kriterien gewählt bzw. die hier benutzten Kriterien ergänzt werden können (z.B. aus anderen Lehrplänen).

Bei der Untersuchung wurden nur zwei voneinander grundsätzlich verschiedene Unterrichtsmethoden betrachtet: die traditionelle und die problemlösende Methode. Wegen der verschiedenen möglichen Vorstellungen über diese Methoden, sind die charakteristischen Merkmale beider Methoden durch folgende Beschreibung festgelegt.

Die als "traditioneller Unterricht" bezeichnete Methode bedeutet kurz zusammengefasst:

eher Frontalunterricht;

leistungsstärkere Schülerinnen und Schüler können manchmal Zusatzaufgaben bekommen, ansonsten arbeiten die Schülerinnen und Schüler an denselben Aufgaben und die Resultate, Lösungen werden gemeinsam besprochen.

Zuerst werden Informationen mitgeteilt bzw. erarbeitet im Unterrichtsgespräch (Begriffe, Definitionen, Theoreme usw.). Es folgt das Einüben, Vertiefen des Erkannten (zumeist über mehrere Stunden). Die verschiedenen Aufgaben werden zu diesem Zweck zusammengestellt, wobei die Aufgaben im Allgemeinen in keiner Beziehung zueinander stehen, sondern einzeln verschiedene (besonders an den Stoff gebundene) Ziele abdecken. Die Lösungen der Aufgaben können leicht als „,richtig“ oder „falsch“ bewertet werden, da für die Schülerinnen und Schüler wenig Freiraum für eigene Initiative erlaubt wird.

Unter "problemlösender Unterricht" wird hier jene Unterrichtsmethode verstanden, die charakterisiert ist durch:

manchmal Frontalunterricht;

die Kinder arbeiten oft paarweise oder in nach verschiedenen Gesichtspunkten organisierten Gruppen (die Organisation hängt von der jeweiligen Aufgabe und von dem gewollten Effekt ab z.B. nach dem Leistungsvermögen der SchülerInnen).

Auch hier werden Bezeichnungen, Definitionen, ... als Informationen mitgeteilt, jedoch werden Theoreme und Begriffe in Probleme eingebettet erarbeitet. Die Aufgaben werden dafür in „Problemgruppen“ gestellt und 
stehen miteinander in Verbindung. Für eigene Initiativen der Schülerinnen und Schüler wird Freiraum angeboten; der Lösungsweg wird von den Schülern und Schülerinnen argumentiert.

Bei den Arbeitsblättern, die theoretisch nach bestimmten Gesichtspunkten konstruiert sind, stellt sich die Frage, inwieweit diese dem ursprünglichen Ziel entsprechen, d.h. inwieweit aus den Blättern die zuvor angegebene Methodencharakteristik herauszulesen ist.

Dies kann z.B. so überprüft werden, dass Personen, die die Konstruktionsmerkmale der Blätter nicht kennen, die Arbeitsblätter bearbeiten, und dann die Methode beschreiben (die Lehrereinstellung), wonach ihrer Meinung nach diese Blätter konzipiert wurden.

Ich habe diese Überprüfung mit ungarischen Lehramtsstudenten durchgeführt.

Die so erhaltenen Bemerkungen stimmen inhaltlich mit den angegebenen Merkmalen zu den beiden Methoden überein.

Hier ist anzumerken, dass nach den Lehramtsstudenten das traditionelle Blatt nicht zur Begriffsbildung geeignet ist. Neben möglichen inhaltlichen Gründen, die hier nicht betrachtet werden, kann dies auch so aufgefasst werden, dass bei einem traditionellen Unterricht es nicht üblich ist, bei begriffseinführenden Stunden mit Arbeitsblättern zu arbeiten. Zuerst werden die Kenntnisse gelernt, dann geübt, und zu dieser Übung werden Aufgaben verwendet. Es ist auch zu bemerken, dass ein (problemlösendes) Arbeitsblatt, wobei die Aufgaben mehrseitig miteinander in Zusammenhang stehen, eher zu einer Begriffsbildung passt, (vgl. Ambrus [1]).

Im weiteren wird ein Unterrichtsinhalt in den beiden Methoden vorgestellt in Form von Arbeitsblättern.

Das Thema ist „Bruchbegriff“ (Begriffsbildung) in der 5. Klasse.

Für den Katalog der Grundtätigkeiten: siehe Lehrplan 2000 [17].

Die im ungarischen Lehrplan (NAT) [11] angegebenen „Allgemeinen Erfordernisse" werden nur kurz zitiert, wie dies im NAT üblich ist. Es handelt sich bei diesem Beispiel um die folgenden Erfordernisse:

1a Anwendung des tätigkeitsgebundenen Zahlenbegriffs.

1e Entwicklung geometrischen Betrachtens in der Ebene und im Raum mit Hilfe geometrischer Modelle.

3b Entscheiden der Gültigkeit einfacher mathematischer und alltäglicher Aussagen. 
3c Gruppieren, Einordnen nach gegebenem oder selbstgewähltem Kriterium, Reihenfolge weniger Elemente, Auswahl nach gegebenen Eigenschaften.

4c Anwenden der Mutter- bzw. Fachsprache mit entsprechender Genauigkeit.

4d Werkzeugartiges Anwenden von verstandenen und gelernten Begriffen und Verfahren.

4g Argumentieren, Widerlegen.

\section{2. Über die Arbeitsblätter des Beispiels}

Die schon erworbenen und die noch zu erwerbenden Kenntnisse sowie die schon erfüllten und noch zu erfüllenden Erfordernisse aus dem ungarischen Lehrplan (NAT) sind die folgenden:

Erfordernisse am Ende der 4. Klasse (NAT):

Anschauliche Begründung des Bruchbegriffes. Ablesen von Bruchzahlen als Messergebnisse, Veranschaulichung von Bruchzahlen mit angegebenen oder gewählten Einheiten: Schätzen, Veranschaulichung von negativen Zahlen anhand verschiedener Modelle. Allgemeine Erfordernisse: 1a, 4a.

Erfordernisse am Ende der 6. Klasse (NAT):

Bruchbegriff anhand anschaulicher Begründung, Allgemeine Erfordernisse: 1a. Die SchülerInnen können in einfachen Fällen Bruchteile von Figuren markieren, Bruchteile von Objekten benennen.

In der 5. Klasse werden zwei Interpretationen des Bruches unterrichtet; die SchülerInnen werden in einfachen Fällen Brüche vergleichen, addieren und subtrahieren, Brüche mit positiven ganzen Zahlen multiplizieren und durch positive ganze Zahlen dividieren.

Die Arbeitsblätter für die 5. Klasse gehen von elementaren Kenntnissen bei Brüchen aus. Bei den geometrischen Figuren, bei denen zum Lösen Ergänzungen, Zerlegungen gemacht werden, kommt das Üben elementarer geometrischer Kenntnisse auch vor. Die SchülerInnen sind schon in früheren Jahren Aufgaben ähnlicher Art (Teilen, Ergänzen, Einordnen, Kuckuckseiersuchen, ... ) begegnet (ganzheitlicher Anspruch).

Es hängt von den SchülerInnen ab, ob sie Papier und Schere zum Lösen der Arbeitsblätter benutzen (dies soll angeboten werden).

Die SchülerInnen können einzeln oder in Gruppen (besonders paarweise) arbeiten. 
Die gemeinsame Besprechung folgt nach der Lösung einer (oder zwei, drei) Aufgaben. Nach Erfahrungen kann die Besprechung nur in leistungsstärkeren Klassen nach der Lösung eines (ganzen) Arbeitsblattes durchgeführt werden.

In der Analyse werden auch Erfahrungen mit ungarischen SchülerInnen angegeben. Wir haben die Arbeitsblätter in einer nicht leistungsstarken Klasse ausprobiert, die sich vorher schon einige Stunden mit Brüchen beschäftigt hat. Für die selbständige Lösung der Arbeitsblätter hatten die SchülerInnen etwa 40 Minuten zur Verfügung. Für diese Klasse war die Anzahl der Aufgaben hoch und einige Aufgaben (besonders einige des problemlösenden Arbeitsblattes) ungewöhnlich. Eine Ursache dafür ist, dass diese Klasse eher traditionell unterrichtet wurde.

Das traditionelle Arbeitsblatt wurde nach dem Lehrbuch (Mathematik 5) von Hajdú [7] aufgebaut.

Bei dem problemlösenden Arbeitsblatt haben wir viele Ideen aus den Arbeitsblättern von Éva Vásárhelyi geschöpft (vgl. Herber/Vásárhelyi [8]).

Die Punktreihen (...) bedeuten „genug Platz für Schülerlösungen“.

\subsection{Arbeitsblätter und zugehörige Kommentare}

Arbeitsblatt (traditionell)

1. Du hast gleichgroße Kreise. Die Kreise bedeuten eine Einheit. Färbe der Reihe nach in den Kreisen: ein Viertel, ein Achtel, drei Achtel, ein Drittel!
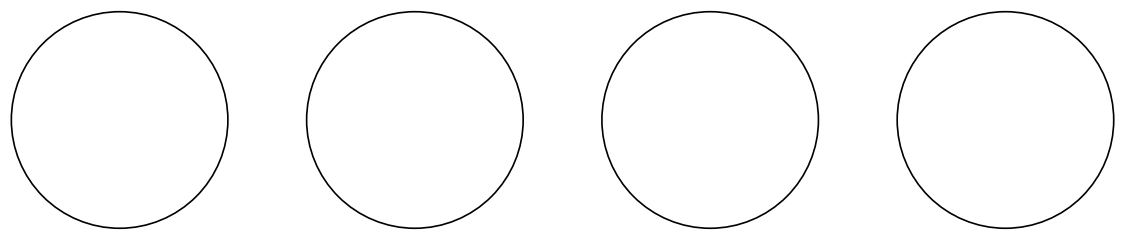

Béla behauptet, dass er ein Sechstel markiert hat. Hat er recht?

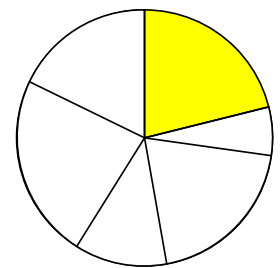


2. Färbe drei Viertel der Sterne rot ein! Wieviel rote Sterne hast du jetzt? Kannst du sagen, welcher Teil der Sterne nicht gefärbt ist?

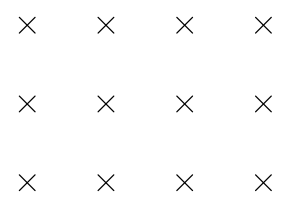

Wir bezeichnen mit $\frac{3}{4}$ (drei Viertel), dass wir „ein Viertel“ dreimal genommen haben. Übliche Benennungen bei Brüchen:

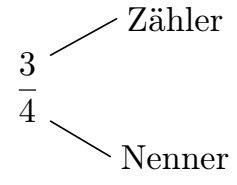

3. Man kann Brüche auch durch Strecken veranschaulichen:

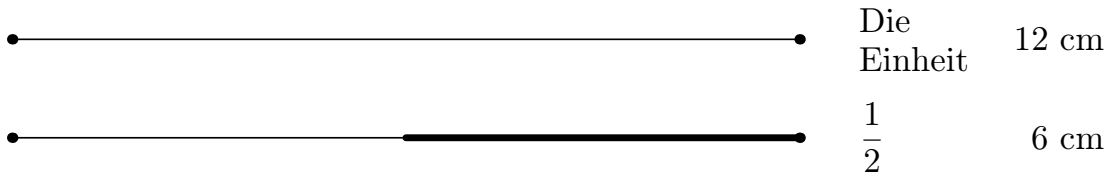

Markiere die angegebenen Teile an den folgenden $(12 \mathrm{~cm}$ langen) Strecken:

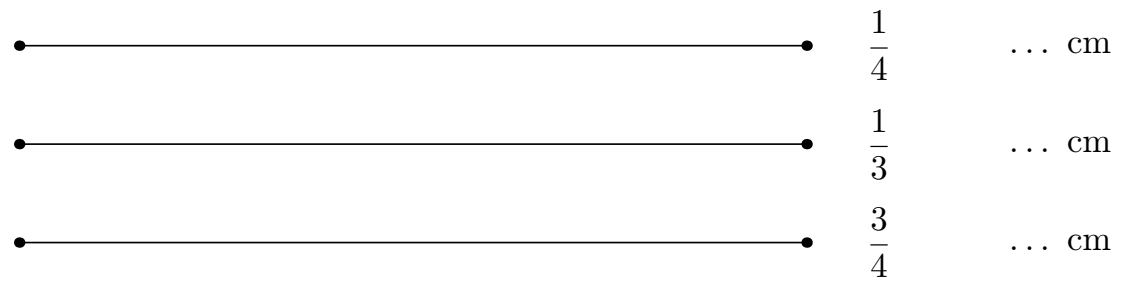

4. Färbe den angegebenen Teil ein! Die Figuren bedeuten je eine Einheit.

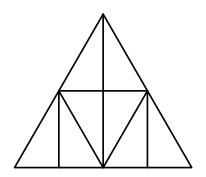

$\frac{1}{4}$

a)

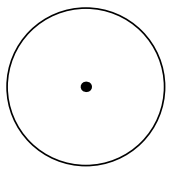

$\frac{3}{4}$

b)

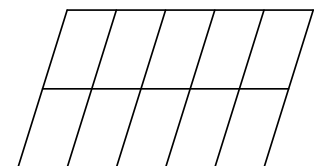

$\frac{3}{10}$

c)

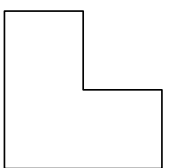

$\frac{3}{4}$

d)

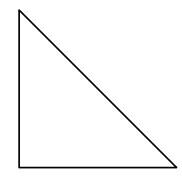

$\frac{1}{2}$

e) 
5. Welcher Teil ist markiert? Die Figuren bedeuten eine Einheit.

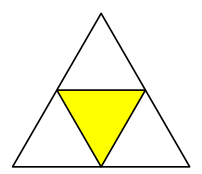

a)

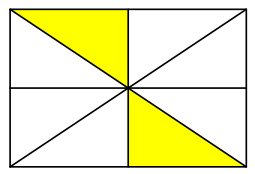

b)

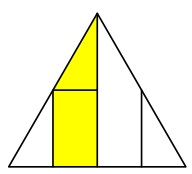

c)

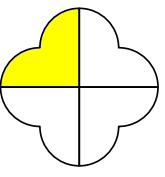

d)

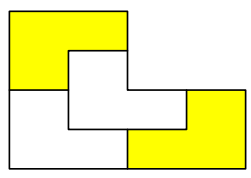

e)

6. Zeichne eine Strecke von $15 \mathrm{~cm}$ ! Dies ist die Einheit. Färbe $\frac{1}{3}$ rot, $\frac{3}{5}$ blau!

7. Am Sonntag haben $\frac{3}{4}$ einer Klasse an einem Ausflug teilgenommen. Wie viele "Schülerinnen" und Schüler haben teilgenommen, wenn die Klasse aus 32 SchülerInnen besteht? (Du kannst auch zeichnen.)

\section{Analyse des Arbeitsblattes}

Die ersten zwei Aufgaben dienen zu einer kurzen Wiederholung und zur Einführung der neuen Benennungen und deren Erklärung. Es werden Bruchteile von stetigen Mengen bei Aufgabe 1 und Bruchteile von diskreten Mengen bei Aufgabe 2 vorgelegt. Wichtig ist bewusst zu machen, dass nur gleichgroße Teile des Ganzen „Bruchteile“ sind.

\section{Erwartungen:}

Ähnliche Aufgaben haben die Kinder schon in der 4. Klasse gelöst; nur bei wenigen SchülerInnen sind Schwierigkeiten zu erwarten. Ein gemeinsames Besprechen der richtigen (schlechten) Lösungen kann fehlerhafte Vorstellungen korrigieren.

Z. B. „Béla hat kein Sechstel markiert, da der Kreis in ungleiche Teile aufgeteilt ist." (Falsche Begründung)

„Béla hat kein Sechstel markiert, denn wenn ich diesen Teil sechsmal nehme, ist das zusammen mehr als der Kreis." (Richtige Begründung)

Üben:

Üben ist hier Wiederholen von vorigen Aufgabentypen mit Brüchen. Aus den Grundtätigkeiten wird ein ,geometrisch-zeichnerisches Darstellen von Objekten“ geübt, aus den Allgemeinen Erfordernissen: 1a, $3 \mathrm{~b}$. 
Die dritte Aufgabe bedeutet einen „Modellwechsel“ (die Kinder müssen Bruchteile an verschiedenen Figuren erkennen). Dieses Modell bereitet das Arbeiten an der Zahlengeraden vor. Nach einem Musterbeispiel werden ähnliche Übungen gemacht. Die Kinder brauchen die vorangehende Information über Benennungen nicht zum Lösen. Dies wäre z.B. dann der Fall, wenn die Kinder die Strecken in so viele Teile schneiden sollen, wie die Anzahl der Nenner angibt.

Die Aufgabe ist hier nach dem Musterbeispiel zu lösen.

Erwartungen:

Bei den Kindern ist zu erwarten, dass diese zuerst die Bruchteile in cm berechnen und auf die $12 \mathrm{~cm}$ langen Strecke ,auftragen“. Wenn die Länge der Strecke nicht angegeben wäre, so ginge es wieder um ein Aufteilen einer Figur.

Der Lehrer kann darauf hinweisen, dass es verschiedene richtige Lösungen gibt. Zum Beispiel zu , $\frac{1}{3}$ ":

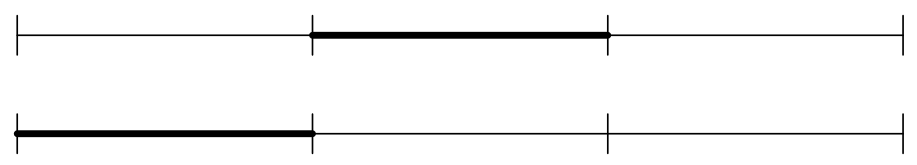

Üben:

Die Aufgabe dient zum Einüben der Bezeichnung des Bruches. Wenn Lösungen verglichen werden, dann werden aus den mathematischen Grundtätigkeiten „Überprüfen von Ergebnissen“ und „geometrisch-zeichnerisches Darstellen von Objekten" geübt, aus den Allgemeinen Erfordernissen: 1a, 4d.

Bei der vierten Aufgabe geht es wieder um einen Modellwechsel und eine Erweiterung auf andere Nenner, aber die Aktivität ist wie vorher: Einfärben von Bruchteilen an Figuren. Bei einigen Aufgaben wird Hilfe zur Lösung durch ein Gitter angeboten. Ähnliche Aufgaben gab es schon in der vierten Klasse.

\section{Erwartungen:}

Hier können schon z.B. Flüchtigkeitsfehler auftreten. Die SchülerInnen bestimmen die Anzahl der Teile fehlerhaft, z.B. bei a). Bei d) können Schwierigkeiten auftreten. In diesem Fall kann der Lehrer bei der Lösung helfen z.B. durch Angeben von weiteren Strecken/Gitter (in persönlichen Gesprächen mit den betreffenden SchülerInnen, während die anderen noch arbeiten). Zu d) kann auch 5 e) Ideen liefern. 
Nach den Erfahrungen mit dieser Aufgabe unter ungarischen SchülerInnen können auch folgende Lösungen vorkommen:

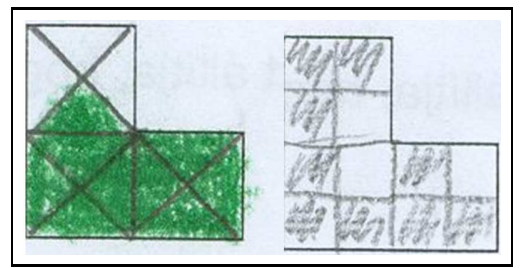

Abbildung 2

Die meisten Schüler haben aber Probleme mit der Lösung und erfahren die richtige Lösung - nach erfolglosem Suchen - in der gemeinsamen Besprechung.

Üben:

Es werden besonders frühere Aufgabentypen mit Brüchen geübt, wobei die eventuell neue Schreibweise verwendet wird; aus den Grundtätigkeiten werden „Anwenden bekannter Verfahren in teilweise neuartiger Situation“, „Geometrischzeichnerisches Darstellen von Objekten“ geübt, aus den Allgemeinen Erfordernissen: $1 \mathrm{a}, 4 \mathrm{~d}$.

Die fünfte Aufgabe ist eine Umkehraufgabe zur Aufgabe 4. Hier müssen die Kinder die Bruchteile aufschreiben. Auch hier gibt es geometrische Inhalte: Zerlegungen und Ergänzungen bereiten auf die (späteren) Flächeninhaltberechnungen vor, ebenso werden Überlegungen über Symmetrien angestellt.

Erwartungen:

Diese Art von Aufgaben ist nicht unbekannt für die SchülerInnen. Bei c) müssen sie vielleicht mehr nachdenken. Die SchülerInnen, die die Bruchteile noch nicht als gleiche Teile auffassen, werden hier $\frac{1}{4}$ oder $\frac{1}{5}$ angeben.

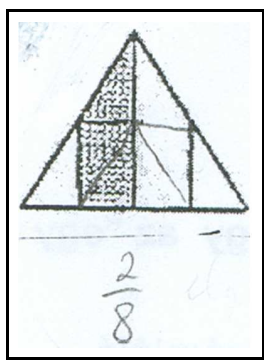

Abbildung 3 
Hier ist es besonders wichtig nachzufragen, wie die SchülerInnen auf gute oder schlechte Lösungen kamen. Die Antworten können so nicht nur verschiedene Lösungswege erklären, sondern auch auf einige Fehler aufmerksam machen.

Falsch gerechnet, oder? Üben:

Hier wird Geometrie (z.B. die Namen von Figuren) und Denkoperationen (Vergleichen, Analysieren, ...) und die bei Aufgabe 4 angeführten Grundtätigkeiten geübt, aus den Allgemeinen Erfordernissen: 1a, 4d, 1e.

Die sechste Aufgabe stellt eine weitere Übung zu Aufgabe 3 dar: auch die Länge der Bruchteile ist anzugeben.

\section{Erwartungen:}

Die SchülerInnen können an der Strecke die Bruchteile aneinander gefügt oder „getrennt“ angeben. Bei der gemeinsamen Besprechung kann der Lehrer (oder die SchülerInnen) die verschiedenen Positionen (Lösungen) der genannten Teile an verschiedenen Strecken aufschreiben. In diesem Fall ist auch zu sehen, dass $\frac{1}{3}$ und $\frac{3}{5}$ zusammen weniger als 1 sind. (Einige SchülerInnen werden dies bemerken).

Üben:

Einüben zu Aufgabe 3. Aus den mathematischen Grundtätigkeiten wird besonders „geometrisch-zeichnerisches Darstellen“ geübt, aus den Allgemeinen Erfordernissen: 1a, 4d.

Die siebente Aufgabe ist eine Weiterführung der Aufgabe 2. Man kann nachfragen, wie viele Kinder an dem Ausflug nicht teilgenommen haben, und welcher Teil der Klasse das war. (Die Frage, „Wie viele Kinder sind zu Hause geblieben?“ ist ungenau und kann eventuell überflüssige Diskussionen auslösen.)

\section{Erwartungen:}

Nur wenige Kinder werden hier Schwierigkeiten haben. Üben:

Üben als Umgehen mit dem Bruchteil einer diskreten Menge. Berechnen des Bruchteiles durch Analogiebildung nach dem Muster (vgl. Parisot [12]).

Die ganze Klasse verhält sich zu $\frac{3}{4}$ der Klasse, wie 32 Schüler zu — Schülern. Oder

$$
1: \frac{3}{4}=32:-
$$


Aus den Grundtätigkeiten wird geometrisch-zeichnerisches Darstellen geübt, aus den Allgemeinen Erfordernissen 1a.

Arbeitsblatt (problemlösend)

1. Du hast gleichgroße Rechtecke. Färbe in verschiedener Weise die Teile:

eine Hälfte
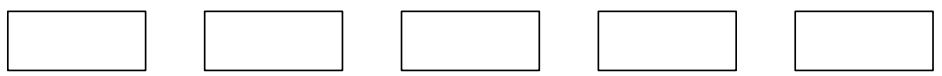

ein Viertel
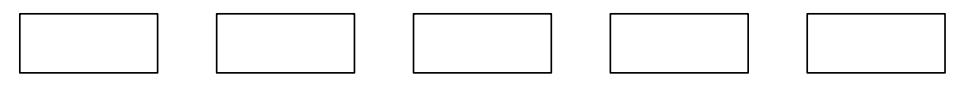

ein Achtel
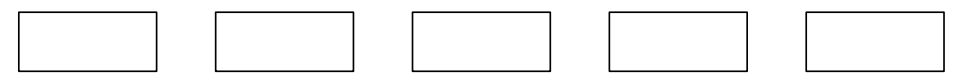

Eine Hälfte schreibt man kurz so: $\frac{1}{2}$ (1 ist geteilt durch 2). Diese Bezeichnung bedeutet, dass das Ganze in zwei gleiche Teile aufgeteilt wurde, und von den zwei Teilen haben wir einen genommen.

Wie würdest du die anderen Bruchteile aufschreiben?

2. Suche nach mehreren Lösungen unter den Brüchen!

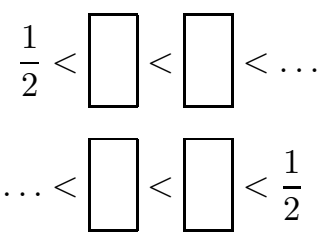

3. Kuckucksei suchen!

a)

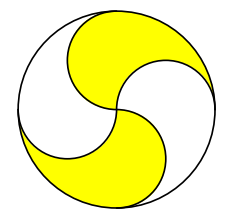

b)

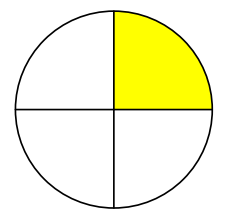

c)

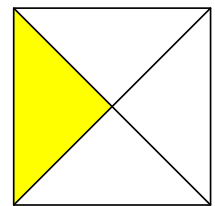

d)

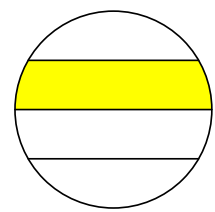

Begründe deine Wahl!

4. Finde zwei weitere Figuren, die in die Reihe passen. 
a)

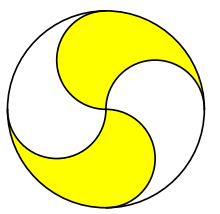

b)

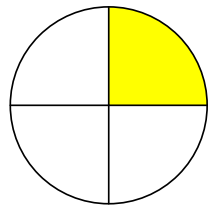

c)

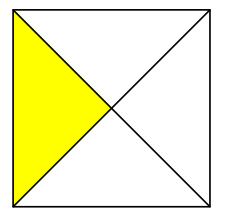

Warum hast du diese Figuren angegeben?

Könntest du auch andersartige Lösungen finden?

5. Kannst du angeben, welcher Anteil markiert ist, wenn jede Figur eine Einheit bedeutet?

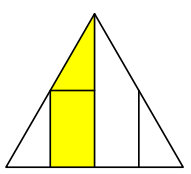

a)

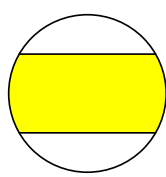

b)

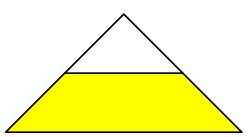

c)

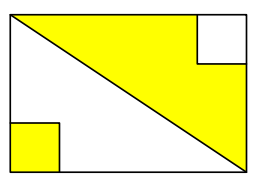

d)

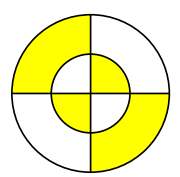

e)

\section{Analyse des Arbeitsblattes}

Bei der ersten Aufgabe macht das Sammeln von verschiedenen Lösungen nicht nur die Aufgabe interessanter, sondern es verlangt sowohl mehr Kreativität als auch das Überblicken verschiedener Lösungsmöglichkeiten (elementare Kombinatorik). Verschiedene, voneinander anscheinend weit liegende Gebiete der Mathematik im Unterricht werden miteinander verbunden; dadurch wird ein möglichst einheitliches Bild über Mathematik geschaffen (Varga [13]). Alle möglichen Lösungen können hier nicht aufgelistet werden (wegen der Vielfalt der Möglichkeiten und der unerwartet auftretenden Lösungen).

Mehrere Rechtecke in einer Reihe sollen zu mehreren Lösungen einer Aufgabe anregen.

Die SchülerInnen können dabei in Gruppen arbeiten und die Ergebnisse gemeinsam besprechen. 
Erwartungen:

Neben den konventionellen Lösungen wie z.B.
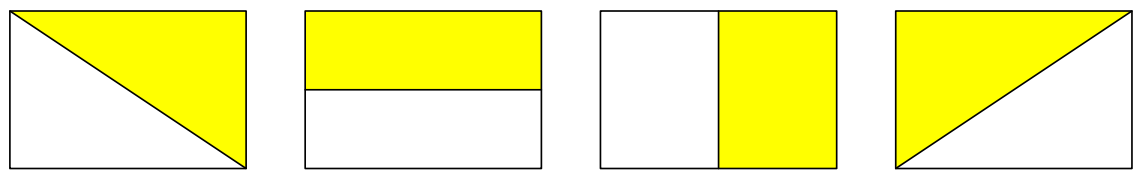

Abbildung 4

können auch solche aufscheinen (bzw. angeregt werden):
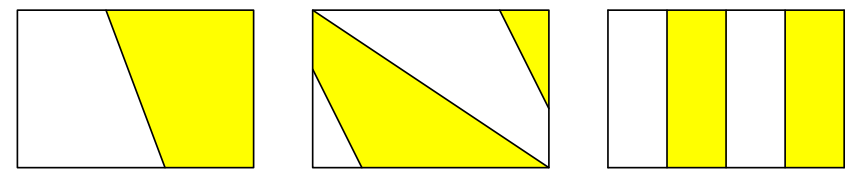

Abbildung 5

Das Vorkommen von anderen Lösungen hängt natürlich von der Klasse und dem vorangehenden Unterricht ab. Ein interessantes Ergebnis ist es, wenn SchülerInnen entdecken, dass es hier unendlich viele Lösungen gibt. Es muss aber nicht forciert werden.

Nach einer möglichen anderen Auffassung der Aufgabe werden die fünf Rechtecke als eine Einheit betrachtet. Nach den Erfahrungen kam diese Möglichkeit oft vor bei den SchülerInnen, nie wurden aber beide Auffassungen betrachtet.

Üben:

Als Sachverhalt werden elementare Kenntnisse über Brüche geübt, bzw. wiederholt. Von den mathematischen Grundtätigkeiten werden „Kombinieren vertrauter Methoden“ (Zerlegung, Ergänzung), „Darstellung mathematischer Objekte“ (hier das Teilen des Ganzen) besonders geübt; von den Allgemeinen Erfordernissen: 1a.

Die zweite Aufgabe ist (auch) mit Hilfe der Erfahrungen beim Lösen der ersten Aufgabe lösbar. Bei der Aufgabe 1 gab es nur Stammbrüche, hier geben die Kinder auch andere Brüche an, und zwar, indem sie ihre Erfahrungen und Kenntnisse beim Ordnen nutzen. Dieses Ordnen wird in weiteren Stunden noch weiterbearbeitet und präzisiert. 
Erwartungen:

$$
\begin{aligned}
\text { z.B. } \quad \ldots \frac{1}{16}<\frac{1}{8}<\frac{1}{4}<\frac{1}{2} \quad \text { oder } \quad \frac{1}{2}<\frac{2}{2}<\frac{3}{2} \ldots \\
\ldots \frac{1}{8}<\frac{1}{6}<\frac{1}{4}<\frac{1}{2} \quad \text { oder } \quad \frac{1}{2}<\frac{4}{2}<\frac{6}{2} \ldots
\end{aligned}
$$

Bei eventuellen Schwierigkeiten, die aber hier nicht zu erwarten sind, kann der Lehrer z.B. auf Halbieren hinweisen, oder/und das Halbieren an einer Lösung der Aufgabe 1 (Hälfte) an Rechtecken durchführen lassen. Es ist hier wichtig, dass die Kinder auch solche Begründungen formulieren, wie „, wenn man die Teile halbiert, werden die so entstandenen Teile immer kleiner" usw.

Hier ist leicht zu erlernen, dass es unendlich viele Lösungen gibt. Die SchülerInnen sollen dafür auch eine Erklärung formulieren.

\section{Üben:}

Aufschreiben und Vergleichen von Brüchen wird geübt. Von den mathematischen Grundtätigkeiten „Anwenden vertrauter Methoden in teilweise neuartigen Situationen" (Ordnen von Brüchen mit der neuen Schreibweise), „Begründen“ (z.B. warum es unendlich viele Lösungen gibt), von den Allgemeinen Erfordernisse: $1 \mathrm{a}, 4 \mathrm{~g}$.

In der dritten Aufgabe können die SchülerInnen mehrere Lösungen (nicht nur in Zusammenhang mit Brüchen) angeben, und es ist auch wichtig, verschiedene Lösungen zu sammeln. Es ist nicht üblich, in mathematischen Aufgaben „Kuckucksei“ zu suchen, obwohl solche Aufgaben gute Möglichkeiten auch für Diskussionen anbieten, und auch motivierend wirken können. Für die meisten Kinder ist d) eine neue Situation (siehe noch Aufgabe 5).

\section{Erwartungen:}

Mögliche richtige Antworten z.B.:

- zu c) hier gibt es keinen Kreis

- zu d) hier ist der gefärbte Anteil nicht bestimmbar

- zu d) es ist unklar (in der 5. Klasse), ob hier der Kreis auf gleiche Teile geteilt wurde

- zu a) hier ist die Figur nicht mit Strecken aufgeteilt. 
Die SchülerInnen müssen ihre Lösung begründen, wodurch entschieden werden kann, ob sie akzeptabel ist.

$\mathrm{Zu} \mathrm{d)} \mathrm{hier} \mathrm{ist} \mathrm{„kein“} \mathrm{Viertel} \mathrm{gefärbt,} \mathrm{da} \mathrm{es} \mathrm{um} \mathrm{ein} \mathrm{Verteilen} \mathrm{auf} \mathrm{ungleiche}$ Teile geht; ist richtig.

$\mathrm{Zu}$ a) hier ist kein Viertel gefärbt aber bei den Anderen ja. Unrichtig, denn es wurde fälschlich angenommen, dass bei d) ein Viertel gefärbt ist.

Bei der Besprechung kann man hinweisen, dass die in d) gefärbten und nicht gefärbten Teile vergleichbar sind: die zwei nicht gefärbten Teile (am Rande) sind gleich groß, ebenfalls die zwei Teile in der Mitte. Ein nicht gefärbter Teil am Rande ist aber kleiner als der gefärbte Teil.

Lösungen mit schlechten Begründungen können abgelehnt oder verbessert werden.

Üben:

Es geht hier um Üben des Erkennens von Bruchteilen an Figuren (teilweise als Wiederholen). Außerdem werden die Grundtätigkeiten „Begründen“, „Analysieren von Begründungen“, „Arbeiten unter bewusster Verwendung von Regeln“ (z.B. Bruchteile bilden), „Überprüfen von Vermutungen“ geübt und von den Allgemeinen Erfordernissen: 1a, 4g, 3b, 3c, 4c.

In der vierten Aufgabe geht es um eine weitere Reihenbildung, wobei die SchülerInnen die Regel selbst finden können. Die Vielfalt der Lösungen ist unabsehbar, aber diese werden zumeist mit Bruchteilen angegeben. Mögliche Lösungen zu finden, erfordert Kreativität und kann auch motivierend wirken. Das Vergleichen der Lösungen kann wieder Diskussionen anregen. Interessante Lösungen können auf geometrische Betrachtungen führen.

\section{Erwartungen:}

Es können mindestens zwei Lösungstypen erwartet werden:

(1) Die Grundgebilde und die Zerlegung bleiben, und der schraffierte Teil wird umgedreht. Aus dem Gesichtspunkt der Drehsymmetrie sind jene Lösungen interessant, die mit der Drehung um $2 \pi / n$ zusammenhängen, denn hier können auch die Regelmäßigkeiten der Drehungen entdeckt werden.

(2) Änderung der Grundgebilde. Dies kann zum Beispiel Kreis $\leftrightarrow$ Quadrat sein (Verschiebung, bzw. Spiegelung der Reihe des originalen Trippels). Die innere Figur wird wiederholt oder nach eigener Regel gewählt. Um die eigene Regel kritisch betrachten zu können, ist es sinnvoll, die Aufgabe für die eigene Figurenserie wiederholen zu lassen. 
Die zitierten Lösungen und die dazugehörigen Erklärungen sind aus den zahlreichen möglichen Lösungen nur einige, die von den Kindern angegeben werden. Der Lehrer kann sehen, ob Bilder und Erklärungen bei den Kindern übereinstimmen, indem er nach einem weiteren Glied fragt.

Einige Lösungen können eventuell durch Beispiele auch von dem Lehrer angeregt werden, aber es ist nicht unbedingt notwendig, auch wenn die Kinder bei einfacheren Lösungen bleiben.

Nach den Erfahrungen ist diese Aufgabe für nicht leistungsstarke SchülerInnen zu schwer, obwohl schon das einfache Wiederholen des originalen Tripels eine richtige Lösung gibt. . .

Üben:

Hier wird das Bruchteilbilden in einer anderen Form geübt und dabei das Vergleichen von geometrischen Objekten mitgeübt. Von den mathematischen Grundtätigkeiten werden besonders: „Begründen“, „,Geometrisch-zeichnerisches Darstellen“ (die SchülerInnen haben die Figuren „im Ganzen“ darzustellen), „Anwenden bekannter Verfahren in teilweise neuartigen Situationen" (Bruchteile-bilden nach selbstgemachter Regel), „Rechtfertigen von Entscheidungen“ (welche Objekte können dazu passen), "Geometrisch-zeichnerisches Darstellen von Objekten“ geübt, von den Allgemeinen Erfordernissen: 3c, 4c.

Für die fünfte Aufgabe haben die Kinder schon (hier und früher) viele Erfahrungen gesammelt, die helfen können. Die bei a) anscheinend überflüssig angegebene Strecke kann helfen.

Die Aufgabe dient auch als Vorbereitung zum Messen des Flächeninhaltes (Zerlegungsgleichheit, Messen mit Hilfe von „Einheitsbereichen“). Um die Bruchteile geometrischer Objekte darstellen zu können, ist die Symmetrie einzelner Figuren (zumindest intuitiv) zu erkennen. Z.B.: kann Achsensymmetrie bei a), Zentralsymmetrie bei d) helfen, oder Drehsymmetrie bei e) für den inneren Kreis bzw. für den Ring.

Die Formulierung als Frage will andeuten, dass diese Aufgabe nicht unbedingt immer lösbar ist. Es ist wichtig, manchmal auch solche Fragen zu stellen, die nicht beantwortbar sind, denn es kann zu „gekünstelt" wirken, wenn nur beantwortbare Fragen in der Schule vorkommen. Es ist wichtig auch solche Probleme zu zeigen, die noch überhaupt nicht beantwortet werden können.

Ich halte diesen Aspekt der Aufgabe auch darum wichtig, weil das in der Schule vermittelte Bild der Mathematik oft dem Grundsatz „Mathematik ist kein fertiges Produkt" widerspricht. 
Erwartungen:

Bei a) ist zu erwarten, dass die SchülerInnen erkennen, dass das kleine Dreieck eine Schlüsselposition hat. Gegebenenfalls sind weitere Hilfsstrecken einzuzeichnen.

Die Lösung hängt aber auch davon ab, welche vorigen Kenntnisse die Kinder mit „Zerlegungen von Figuren“ haben, die hier der Lehrer aktivieren kann. Nach der richtigen Antwort und Begründung kann der Lehrer noch nach weiteren Begründungen fragen und/oder solche selbst zeigen.

\begin{tabular}{|c|c|c|}
\hline$\frac{3}{8}$ ist gefärbt & Begründung & Spätere Anwendung \\
\hline & Zerlegungsgleich & $\begin{array}{l}\frac{1}{8} \text { fehlt zu einem halben Dreieck }\left(\frac{4}{8}\right) \\
\frac{3}{8}+-\overline{-}=\frac{4}{8} \\
\frac{4}{8}-\frac{3}{8}=- \\
\frac{1}{2}-\frac{1}{8}=\end{array}$ \\
\hline & $\begin{array}{l}\text { Andere } \\
\text { Zerlegungsgleichheit }\end{array}$ & $\begin{array}{l}\frac{1}{8} \text { fehlt aus einer Hälfte (die } \frac{4}{8} \text { ist) } \\
\frac{3}{8}+{ }_{-}=\frac{1}{2} \\
\frac{1}{2}-\frac{3}{8}= \\
\frac{1}{2}-\frac{1}{8}=\end{array}$ \\
\hline & $\begin{array}{l}\text { Zerlegungsgleich } \\
\text { wieder anders }\end{array}$ & $\frac{2}{8}+\frac{1}{8}=$ \\
\hline & $\begin{array}{l}3 \text { der } 4 \text { Teil-Dreiecke } \\
\text { werden halbiert }\end{array}$ & $\frac{3}{4}: 2=$ \\
\hline
\end{tabular}

\section{Abbildung 6}

Die Verwandtschaft von b) mit der entsprechenden Figur der Aufgabe 3 d) ist ohne Zweifel. Die mögliche Antwort hier: nein. Da die Kenntnisse noch nicht ausreichen, können die Kinder den gefragten Anteil nicht genau angeben. 
Bei c) ist wieder ein kleines Dreieck die Schlüsselfigur.

a) und c) sind nicht in gleiche Teile geteilt, können aber so ergänzt werden.

Bei den Aufgaben a), b) c) ist als Schülerlösung zu erwarten: „ich konnte den Anteil nicht angeben, da diese Figuren nicht in gleiche Teile geteilt sind." Von der Situation abhängig kann diese Antwort als richtig oder als noch detailliert auszuführend betrachtet werden.

Bei d) können vorige Erfahrungen (z.B. bei Aufgabe 1) helfen, aber die Lösung durch Ergänzung ist nicht besonders schwer.

e) die Symmetrie der Figur d) hilft. Die gleichfarbigen Teile können zum Halbviertelkreis ergänzt werden.

Nach den Erfahrungen erwies sich die anscheinend richtige Lösung $\frac{4}{8}$ bei den SchülerInnen als falsch, da sie in diesem Fall von ungleichen Teilen 4 genommen haben.

Üben:

Das Erkennen von Bruchteilen und auch geometrische Zerlegungen, Ergänzungen werden geübt sowie von den Grundtätigkeiten: „Kombinieren vertrauter Methoden“, „Begründen“ und von den Allgemeine Erfordernissen: 1e, 4c, 4g.

\section{Methodenabhängigkeit des Übens - anhand des Beispiels}

Bei der Behandlung des Sachverhaltes „Brüche in der 5. Schulstufe“ geht es um einen ganzheitlichen sowohl geometrischen als auch algebraischen Zugang zu einem Begriff. Diese Art der Begriffsbildung macht es besonders interessant, an diesem Beispiel zu untersuchen was, wo und wie geübt wird.

$\mathrm{Zu}$ der vergleichenden Untersuchung sei zuerst angegeben, welche Kenntnisse bei den beiden Arbeitsblättern geübt werden:

Tabelle 1

Traditionelles Arbeitsblatt

Wiederholen früherer Aufgabentypen mit Brüchen

Modellwechsel

Bruchbezeichnung

Geometrie (symmetrische Figuren, Namen von Figuren)

Bruchteile berechnen und darstellen 
Fortsetzung Tabelle 1

\begin{tabular}{|l|}
\hline Problemlösendes Arbeitsblatt \\
\hline Wiederholen von Kenntnissen über Brüche \\
Aufschreiben und Vergleichen von Brüchen \\
Erkennen von Bruchteilen an Figuren, Bruchteile bilden \\
Vergleich von geometrischen Objekten \\
Geometrische Zerlegung, Ergänzung \\
Selbständiges Finden und Begründen von Lösungen \\
\hline
\end{tabular}

Schon die aufgelisteten Kenntnisse zeigen sowohl eine qualitative als auch eine quantitative Verschiedenheit zwischen den beiden Methoden. Bei dem traditionellen Unterricht werden neben dem bereits Erlernten besonders die vorangehenden relevanten Kenntnisse geübt (wiederholt). Beim problemlösenden Unterricht ist das Spektrum breiter. Das Wiederholen von Bruchteilbestimmungen geht in die Richtung „Vorbereitung von geometrischen Transformationen" weiter und kann auch zum Vergleich und Rechnen mit Brüchen mehr Erfahrungen anbieten als im traditionellen Fall (vgl. z.B. Aufgaben I/1-II/1, I/5-II/5). Beim problemlösenden Blatt wird mit dem Vergleich der verschiedenen Lösungswege „Argumentieren“ und „Begründen“ geübt.

Tabelle 2 stellt Grundtätigkeiten beziehungsweise Allgemeine Erfordernisse gegenüber, die anhand der beiden Arbeitsblätter geübt werden und gibt die Anzahl der Aufgaben an, wo diese in den Arbeitsblättern konkret geübt sind:

Tabelle 2

\begin{tabular}{|l|c|c|}
\hline Grundtätigkeiten (Österreichischer Lehrplan) & $\begin{array}{c}\text { Traditionelles } \\
\text { Arbeitsblatt }\end{array}$ & $\begin{array}{c}\text { Problem- } \\
\text { lösendes } \\
\text { Arbeitsblatt }\end{array}$ \\
\hline $\begin{array}{l}\text { Geometrisch-zeichnerisches Darstellen von } \\
\text { Objekten, Arbeiten mit solchen Darstellungen }\end{array}$ & 5 & 3 \\
\hline $\begin{array}{l}\text { Anwendungen bekannter Verfahren in teilweise } \\
\text { neuartiger Situation }\end{array}$ & 2 & 2 \\
\hline Überprüfen von Ergebnissen & 1 & 1 \\
\hline Überprüfen von Vermutungen & - & 1 \\
\hline Kombinieren vertrauter Methoden & - & 2 \\
\hline
\end{tabular}


Fortsetzung Tabelle 2

\begin{tabular}{|l|c|c|}
\hline Grundtätigkeiten (Österreichischer Lehrplan) & $\begin{array}{c}\text { Traditionelles } \\
\text { Arbeitsblatt }\end{array}$ & $\begin{array}{c}\text { Problem- } \\
\text { lösendes } \\
\text { Arbeitsblatt }\end{array}$ \\
\hline Begründen & - & 5 \\
\hline Analysieren von Begründungen & - & 1 \\
\hline Darstellung mathematischer Objekte & - & 2 \\
\hline Arbeiten unter bewusster Verwendung von Regeln & - & 1 \\
\hline Rechtfertigen von Entscheidungen & - & 1 \\
\hline
\end{tabular}

\begin{tabular}{|l|c|c|}
\hline Allgemeine Erfordernisse (Ungarischer Lehrplan) & $\begin{array}{c}\text { Traditionelles } \\
\text { Arbeitsblatt }\end{array}$ & $\begin{array}{c}\text { Problem- } \\
\text { lösendes } \\
\text { Arbeitsblatt }\end{array}$ \\
\hline $1 \mathrm{a}$ (Pflicht) & 6 & 3 \\
\hline $3 \mathrm{~b}$ & 1 & 1 \\
\hline $4 \mathrm{~d}$ & 3 & - \\
\hline $1 \mathrm{e}$ & 1 & 1 \\
\hline $4 \mathrm{~g}$ & - & 3 \\
\hline $3 \mathrm{c}$ & - & 2 \\
\hline $4 \mathrm{c}$ & - & 3 \\
\hline
\end{tabular}

Es ist zu sehen, dass am traditionellen Blatt weniger Grundtätigkeiten und Allgemeine Erfordernisse, aber diese im allgemeinen öfter geübt werden. Dies entspricht der traditionellen Auffassung, wo eher sichere Basiskenntnisse und Fertigkeiten als bewegliches Denken und neuartiges Anwenden von Kenntnissen wichtig sind (vgl. auch die Beschreibung der beiden Methoden).

Die Gewichtung der einzelnen Grundtätigkeiten und Erfordernisse entspricht auch den vorigen Bemerkungen: Bei dem traditionellen Blatt wird „geometrischzeichnerisches Darstellen“ beziehungsweise das Arbeiten mit solchen Darstellungen in so vielen Aufgaben geübt wie beim problemlösenden Blatt „Begründen“. Die Verteilung der „Anzahl“ der Erfordernisse ist beim problemlösenden Blatt gleichmäßiger. Das traditionelle Arbeitsblatt „konzentriert sich“ eher an dem pflichtgemäßen 1a; beim problemlösenden Blatt scheinen andere Erfordernisse genauso wichtig zu sein. 
Die Unterrichtsmethodenabhängigkeit des Übens ist sowohl auf der Ebene der Kenntnisse als auch auf der Ebene der Grundtätigkeiten/Allgemeinen Erfordernisse deutlich zu erkennen.

\section{Methodenabhängigkeit des Übens - zusammenfassende Ergebnisse}

Nach dem vorher ausgeführten Beispiel und der erwähnten weiteren zwei Beispiele in [1] ist festzustellen:

Die Methodenabhängigkeit des Übens ist mit den Kriterien auf der Ebene der Grundtätigkeiten und der Allgemeinen Erfordernisse gut, bei den Kenntnissen weniger deutlich beschreibbar.

Es können auch Aussagen formuliert werden zum Charakterisieren der Methodenabhängigkeit des Übens.

Tabelle 3

\begin{tabular}{|l|c|c|c|}
\hline & $\begin{array}{c}\text { Bruch- } \\
\text { begriff }\end{array}$ & $\begin{array}{c}\text { Beispiel } \\
2\end{array}$ & $\begin{array}{c}\text { Beispiel } \\
3\end{array}$ \\
\hline $\begin{array}{l}\text { Die „Anzahl“ der geübten verschiedenen Kenntnisse } \\
\text { ist etwa gleich bei beiden Arbeitsblättern }\end{array}$ & + & + & + \\
\hline $\begin{array}{l}\text { Die „Anzahl“ der geübten verschiedenen Grund- } \\
\text { tätigkeiten ist größer im problemlösenden Fall, }\end{array}$ & + & + & + \\
\hline $\begin{array}{l}\text { Die „Anzahl“ der geübten Allgemeinen Erfordernisse } \\
\text { ist größer im problemlösenden Fall, }\end{array}$ & + & + & - \\
\hline $\begin{array}{l}\text { Im traditionellen Fall gibt es weniger verschiedene } \\
\text { Grundtätigkeiten und Allgemeine Erfordnisse, } \\
\text { diese treten öfter auf }\end{array}$ & + & - & - \\
\hline $\begin{array}{l}\text { Gleichmäßige Verteilung der „Anzahlen“ der } \\
\text { einzelnen Grundtätigkeiten und Allgemeinen } \\
\text { Erfordernisse beim problemlösenden Arbeitsblatt }\end{array}$ & + & - & + \\
\hline $\begin{array}{l}\text { Im traditionellen Fall wird auf pflichtgemäße } \\
\text { Allgemeine Erfordnisse (erkennbar durch größere } \\
\text { „Anzahl“) konzentriert }\end{array}$ & + & - & + \\
\hline
\end{tabular}

$(+$ trifft $\mathrm{zu},-$ trifft nicht $\mathrm{zu})$ 
Fortsetzung Tabelle 3

\begin{tabular}{|l|c|c|c|}
\hline & $\begin{array}{c}\text { Bruch- } \\
\text { begriff }\end{array}$ & $\begin{array}{c}\text { Beispiel } \\
2\end{array}$ & $\begin{array}{c}\text { Beispiel } \\
3\end{array}$ \\
\hline $\begin{array}{l}\text { Das pflichtgemäße Allgemeine Erfordernis und } \\
\text { „Argumentieren, Widerlegen“ kommen gleich oft vor } \\
\text { im problemlösenden Fall }\end{array}$ & + & + & + \\
\hline $\begin{array}{l}\text { Das pflichtgemäße Allgemeine Erfordernis kommt im } \\
\text { traditionellen Fall öfter vor als ,Argumentieren, } \\
\text { Widerlegen“ }\end{array}$ & - & + & + \\
\hline
\end{tabular}

(+ trifft $\mathrm{zu},-$ trifft nicht $\mathrm{zu})$

So ergeben sich folgende Aussagen, die die beiden Methoden bezüglich Üben charakterisieren:

Bei einem problemlösenden Unterricht:

(1) relativ große „Anzahl“ von Grundtätigkeiten

(2) relativ große „Anzahl“ von Allgemeinen Erfordernissen

(3) das Allgemeine Erfordernis „Argumentieren, Widerlegen“ und die im ungarischen Lehrplan pflichtgemäß vorgeschriebenen Erfordernisse kommen gleich oft vor.

Bei einem traditionellen Unterricht:

(1) relativ kleine „Anzahl“ von Grundtätigkeiten

(2) relativ kleine „Anzahl“ von Allgemeinen Erfordernissen

(3) die im ungarischen Lehrplan pflichtgemäß vorgeschriebenen Erfordernisse kommen bedeutend öfter vor als das Allgemeine Erfordernis „Argumentieren, Widerlegen".

Es ist hier anzumerken, dass obwohl die problemlösende Unterrichtsmethode mehr effektiv erscheint, da mehr Kenntnisse und mehr Elemente aus den Anforderungskatalogen geübt wurden als bei dem traditionellen Unterricht, sind wir der Meinung, dass eben wegen dieser Vielfalt die problemlösende Methode zum Vertiefen und Festigen des Gelernten im allgemeinen nicht ausreicht. (Vgl. „Kompensatorische Aspekte der Hausübung“, Ambrus [1]) 


\section{Weitere Ergebnisse und Ausblick}

Wie dies schon in 3.1 erwähnt wurde, wurde das Üben anhand von vier ungarischen Unterrichtsbeispielen untersucht aus denen hier eines angeführt wurde.

Bei den Beispielen wurde neben der Methodenabhängigkeit des Übens aufgezeigt, dass das Üben - in fallender Reihung- auch von den folgenden Aspekten abhängt: Bereiche der Mathematik, Lernarten, Schulstufe, Medien sowie dass eine Abhängigkeit des Übens auch von verwendeten Werkzeugen, Modellen, Hausübung, Schulbuch und Land (Sprachraum) vorhanden ist.

Nach den verwendeten Kriterien zur Untersuchung des Übens in dieser Arbeit kann eine weitere Ebene für die Komplexität des Übens nach der Anzahl der Kenntnisse, Allgemeinen Erfordernisse und Grundtätigkeiten angegeben werden. Das Ordnen des Übens in „erste, vielfältige, komplexe“ Übungen (inhaltlich auch in der ungarischen Didaktik vorhanden) betrachtet die inhaltliche Dimension des Vorgehens beim Lösungsgang (Schrittanzahl) der (Lehrer-)Musterlösung (komplexe Übungen können für sogenannte Begabte weniger „komplex“ erscheinen).

In ähnlicher Art können andere Aufgabenkriterien bezüglich des Übungseffektes bewertet werden.

Diese Arbeit beschäftigt sich mit der Planung des Übens bei einzelnen Unterrichtsstunden. Die Planung von Unterrichtssequenzen bis hin zur Jahresplanung unter den Aspekten einer vielfältigen Übung sollte Gegenstand einer weiteren Erforschung sein, die auch das entsprechende Aufgabenstellen umfasst. Ebenso ist eine Weiterentwicklung der (Übungs-)Kriterien insbesondere für das Problemlösen und die Einbindung weiterer Lehrplanvorgaben (wie Kompetenzen, Sozialerziehung, ... ) in die Übung anzustreben als- Erweiterung des Bildes vom Mathematikunterricht.

Durch weitere Ländervergleiche sollen die eigenen Bedingungen des Unterrichts reflektiert und internationale Leistungsvergleiche der SchülerInnen (z.B. OECD-Studien) besser interpretierbar werden.

Die Planung und Analyse des Übens hilft bei einer mehr durchdachten und effektiven Unterrichtsgestaltung. Wir halten es daher für wichtig, Fragen der Forschung bzw. Planung des Übens im Mathematikunterricht auch in die Lehrer- aus - und weiterbildung einzubeziehen.

Ich möchte mich herzlich bedanken für die wertvolle Ratschläge bei András Ambrus, bei Ervin Deák und bei den beiden Lektoren. 


\section{Literatur}

[1] G. Ambrus, Üben in der Planung des Mathematikunterrichts, Dissertation, Salzburg, 2003.

[2] H. J. Claus, Einführung in die Didaktik, Wissenschaftliche Buchgesellschaft, Darmstadt, 1989.

[3] Comenius, Didactica Magna, (Übersetzung: Geréb Gy.), Seneca Kiadó, Budapest, 1992.

[4] L. Engelmann and G. Liesenberg, Übung macht den Meister! Schülerorientierte Gestaltung täglicher Übungen, Mathematik in der Schule 9 (1993).

[5] L. Flade and L. Goldberg, Tägliche - erste vielfältige - komplexe Übungen, Mathematiklehren 53 (1992).

[6] L. Flade and M. Pruzina, Bloß eine Übungsstunde, Mathematik in der Schule 5 (1992), 283-289.

[7] S. Hajdú (Hrsg.), Matematika 5, (Lehrbuch für Mathematik 5. Klasse), Műszaki Kiadó, Budapest, 1994.

[8] H.-J. Herber and É. Vásárhelyi, Analogiebildung und Innere Differenzierung, SFB Projekt-Bericht, Salzburg, 1998.

[9] J. Iker, S. Szerencsi and Gy. Vörös, A Matematika Tanítása I., (Hrsg.: Ács P.), Tankönyvkiadó, Budapest, 1991.

[10] H. Maier, Zur Übung im Fach Mathematik, Pädagogische Welt 3 (1986), 103-108.

[11] Nemzeti Alaptanterv - NAT, Müvelődési és Közoktatási Minisztérium, Korona Kiadó, 1995.

[12] K. J. Parisot, H.-J. Herber, H. Astleitner and É. Vásárhelyi, Analogie und Problemlösen, in: Trends im Geometrieunterricht, (Parisot, K. J., Vásárhelyi, É. (Hrsg.)), Abakus Verlag, Salzburg, 1996, 89-90.

[13] T. Varga, A matematika tanítása, [Mathematik Lehren], Tankönyvkiadó, Budapest, 1964.

[14] W. Walsch and W. Jungk, Zum Prozess des Festigens, Sich daraus ergebende Anforderungen an den Unterricht in den Klassen 9, 10 /1, Mathematik in der Schule 6 (1987).

[15] H. Winter, Begriff und Bedeutung des Übens im Mathematikunterricht, Mathematiklehren 2 (1984).

[16] E. Ch. Wittmann, Grundfragen des Mathematikunterrichts, Vieweg, 1981.

[17] 133. Verordnung: Änderung der Verordnung über Lehrpläne der allgemeinbildenden höheren Schulen; Bekanntmachung der Lehrpläne für den Religionsunterricht an diesen Schulen, Bundesgesetzblatt für die Republik Österreich, Jahrgang 2000, Teil II, Ausgegeben am 11. Mai 2000.

GABRIELLA AMBRUS

1136 BUDAPEST HEGEDÜS GY. U. 36

HUNGARY

E-mail: ambrusg@ludens.elte.hu

(Received January, 2005) 